\title{
BMJ Open The CopenHeartSF trial-comprehensive sexual rehabilitation programme for male patients with implantable cardioverter defibrillator or ischaemic heart disease and impaired sexual function: protocol of a randomised clinical trial
}

To cite: Johansen PP, Zwisler A-D, HastrupSvendsen J, et al. The CopenHeartSF trialcomprehensive sexual rehabilitation programme for male patients with implantable cardioverter defibrillator or ischaemic heart disease and impaired sexual function: protocol of a randomised clinical trial. BMJ Open 2013;3:e003967. doi:10.1136/ bmjopen-2013-003967

- Prepublication history for this paper is available online. To view these files please visit the journal online (http://dx.doi.org/10.1136/ bmjopen-2013-003967).

Received 6 September 2013 Revised 9 October 2013 Accepted 10 October 2013

\section{CrossMark}

For numbered affiliations see end of article.

Correspondence to Pernille Palm Johansen; pernille.palm.johansen@ regionh.dk

\section{ABSTRACT}

Introduction: Sexuality is an important part of people's physical and mental health. Patients with heart disease often suffer from sexual dysfunction. Sexual dysfunction has a negative impact on quality of life and well-being in persons with heart disease, and sexual dysfunction is associated with anxiety and depression. Treatment and care possibilities seem to be lacking. Studies indicate that non-pharmacological interventions such as exercise training and psychoeducation possess the potential of reducing sexual dysfunction in patients with heart disease. The CopenHeartSF trial will investigate the effect of a comprehensive sexual rehabilitation programme versus usual care

Methods and analysis: CopenHeartSF is an investigator-initiated randomised clinical superiority trial with blinded outcome assessment, with 1:1 central randomisation to sexual rehabilitation plus usual care versus usual care alone. Based on sample size calculations, 154 male patients with impaired sexual function due to implantable cardioverter defibrillator or ischaemic heart disease will be included from two university hospitals in Denmark. All patients receive usual care and patients allocated to the experimental intervention group follow a 12-week sexual rehabilitation programme consisting of an individualised exercise programme and psychoeducative consultation with a specially trained nurse. The primary outcome is sexual function measured by the International Index of Erectile Function. The secondary outcome measure is psychosocial adjustment to illness by the Psychosocial Adjustment to Illness Scale, sexual domain. A number of explorative analyses will also be conducted.

Ethics and dissemination: CopenHeartSF is approved by the regional ethics committee (no H-42012-168) and the Danish Data Protection Agency (no 2007-58-0015) and is performed in accordance with good clinical practice and the Declaration of Helsinki in its latest form.
Strengths and limitations of this study

The study has been designed to meet the criteria for high quality in non-pharmacological randomised clinical trial with central randomisation, multicentre participation and blinded assessment and analysis

- We are aware of the subjective nature of the selfreported primary outcome (International Index of Erectile Function). Accordingly, we will interpret data conservatively.

- This trial is the first to test the effect of a comprehensive approach on sexual dysfuntion in patients with ischaemic heart disease or implantable defibrillators.

Registration: Clinicaltrials.gov identifier: NCT01796353.

\section{BACKGROUND}

Sexuality is an important part of people's physical and mental health. ${ }^{1}$ Patients with cardiovascular disease have an increased prevalence of sexual dysfunction. ${ }^{3-5}$ The causes of sexual dysfunction can be related to physical changes due to the disease, mental changes or adverse reactions to drugs and other interventions. ${ }^{6} 7$ Male sexual dysfunction is divided into sexual interest/desire disorders, ejaculation and orgasmic dysfunctions and erectile dysfunction. ${ }^{8}$ The most common disorder is erectile dysfunction, defined as the persistent inability to obtain or maintain an erection which enables satisfying sexual activity. ${ }^{9}$ Erectile dysfunction is associated with age, but can also be triggered by organic as well as psychogenic conditions 
and is often related to vascular disease such as diabetes, hypertension and heart disease. ${ }^{10}$ Studies including 33451 males estimate that erectile dysfunction in varying degrees exists in $52 \%$ of all men, and that age is the most common variable associated with erectile dysfunction. ${ }^{3-5}$ The probability of complete erectile dysfunction in cardiovascular patients is $39 \%$ compared to $10 \%$ in the total population when adjusting for age. ${ }^{34}$ Physical activity is positively associated with a lower incidence of erectile dysfunction. ${ }^{5}$ The prevalence of sexual dysfunction in patients with heart disease ranges from $15 \%$ up to $89 \% .^{11-17}$ Patients with ischaemic heart disease and patients with implantable cardioverter defibrillator, which are two large and growing patient populations, are especially affected. ${ }^{11}{ }^{16-20}$ Sexual dysfunction has a negative impact on quality of life and well-being in men with cardiovascular disease and sexual dysfunction is associated with an increase in anxiety and depression. $^{21-24}$ The relationship is perceived to be bidirectional, with one element forcing the other. ${ }^{25} 26$

\section{Standard treatment}

Despite the fact that several international guidelines recommend that health professionals address the topic of sexuality in patients with heart disease, ${ }^{27} 28$ this is rarely practiced. ${ }^{29} 30$ The consensus or practice on how or where patients with heart disease and sexual dysfunction should be treated is lacking, however, some guidelines about the prescription of phosphodiesterase-5 (PDE-5) inhibitors exist. ${ }^{6}$ The PDE-5 inhibitors have an overall success rate of $50-80 \%$ of those treated among patients with cardiovascular disease. ${ }^{6} 31 \quad 32$ PDE-5 inhibitors are generally safe. Linking PDE-5 inhibitors to cardiac events, large randomised trials and a meta-analysis suggest that they are not associated with an increase in myocardial infarction or cardiac events. ${ }^{6}{ }^{32}$ In patients with heart disease and no effect of PDE-5 inhibitors, or where PDE-5 inhibitors are contraindicated because of treatment with nitrates, there seems to be no consensus on what treatment should be offered for sexual dysfunction.

\section{Non-pharmacological treatment potentials}

Non-pharmacological interventions possess potential in reducing sexual dysfunction. Lifestyle factors such as cigarette smoking, hyperlipidaemia and a sedentary lifestyle all predict erectile dysfunction ${ }^{45}$ and these are the same risk factors that predict coronary artery disease. A recent meta-analysis of six randomised trials with 740 patients with no known heart disease, showed that lifestyle modifications such as physical exercise and pharmacotherapy for cardiovascular risk factors were associated with a significant improvement in erectile function. ${ }^{33}$ Furthermore, a randomised trial investigating the effect of exercise training $3 \mathrm{~h} /$ week or more in patients with no heart disease showed a significant result in improving the person's erectile function compared with controls with no exercise training. ${ }^{34}$ We hypothesise that these lifestyle modifications can also improve sexual dysfunction in patients with already established heart disease. A systematic literature search showed five randomised clinical trials which examine the effect of physical exercise on sexual dysfunction. ${ }^{35-39}$ Overall, 591 patients with heart disease were included and the effect was significant in three of the five trials. ${ }^{37-39}$ However, the trials are characterised as being of small sample sizes, using non-validated tools and mainly focusing on the time before patients return to sexual activity and not on the ability and quality of the sexual performance. Randomised trials that address the psychological aspects of sexual dysfunction are limited in patients with heart disease. However, one randomised trial testing the effect of sexual therapy showed some promising trends when it comes to improving the frequency and quality of sexual activity in male patients postcardiac event beyond the usual cardiac rehabilitation. ${ }^{40}$ However, due to the limited power of the sample in this trial, it did not allow the detection of significant effects. The role of pelvic floor exercises as a treatment of erectile dysfunction is not tested on patients with heart disease, but in the general population $40-47 \%$ had regained normal erectile function after 3-4 months of training the pelvic floor muscles. ${ }^{41}{ }^{42}$ As the condition of sexual dysfunction often includes both physical and psychological components, it is plausible to believe that patients with heart disease and sexual dysfunction benefit from a comprehensive rehabilitation intervention ${ }^{43}{ }^{44}$ consisting of a psychoeducational component and an exercise training component including pelvic floor exercises.

\section{TRIAL OBJECTIVES}

The objective of the CopenHeartSF is to investigate benefit and harm on the sexual function of male patients with ischaemic heart disease or patients with implantable cardioverter defibrillator of a comprehensive sexual rehabilitation programme, consisting of a psychoeducative component and a physical exercise component, including pelvic floor exercises. The primary hypothesis is that, a comprehensive sexual rehabilitation programme improves sexual function, as assessed by the International Index of Erectile Function (IIEF) questionnaire ${ }^{45} 46$ in males with sexual dysfunction and ischaemic heart disease or patients with implantable cardioverter by 3.5 points in the experimental group compared with the control group after completion of the programme. The estimated increase in primary outcome is based on a study that examines the effect of a physical intervention in patients with cardiovascular disease taking PDE-5 inhibitors. ${ }^{34}$ The secondary hypothesis is that sexual function, measured by the sexual domain in the Psychosocial Adjustment to Illness Scale (self-reported version; PAIS-SR) questionnaire ${ }^{47}$ improves by two points in the experimental group compared with the control group after completing the programme. The estimated increase in secondary outcome is based on two studies that examine the prevalence of sexual dysfunction in patients with heart failure. ${ }^{48} 49$

Exploratory analyses will test the hypotheses that comprehensive sexual rehabilitation will improve: health-related quality of life, anxiety and depression, frequency of sexual 
activity, physical capacity measured by peak oxygen uptake (peak $\mathrm{VO}_{2}$ ), pelvic floor muscle strength and endurance and female assessment of male partner's erectile dysfunction.

\section{METHODS}

CopenHeartSF is an investigator-initiated randomised clinical superiority trial with blinded outcome assessment, with 1:1 central randomisation to a comprehensive sexual rehabilitation programme plus usual care or usual care alone. Based on sample-size calculations, 154 patients will be recruited from two university hospitals in Denmark. The CopenHeartSF trial is a part of the overall CopenHeart project, consisting of several randomised clinical trials (http://www.CopenHeart.org), designed to develop evidence-based knowledge of rehabilitation among patients with complex cardiac conditions. Major parts of the CopenHeartSF methods section and trial design in this article are similar to other randomised clinical trials, CopenHeartIE, ${ }^{50}$ CopenHeartRFA ${ }^{51}$ and CopenHeartVR. ${ }^{52}$

\section{Study population and eligibility criteria}

Male patients above 18 years with sexual dysfunction associated with implantable cardioverter defibrillator or with ischaemic heart disease verified by coronary angiography, who have a partner, speak and understand Danish, and provide a written informed consent, are considered eligible for participation. Exclusion criteria are patients at intermediate or high risk in relation to their cardiovascular status according to recommendations from the Princeton consensus group ${ }^{32} 53$; those with diseases in the urinary tract; those who perform intense exercise more than $3 \mathrm{~h}$ a week; patients with neurological or orthopaedic deficits which prevent training; patients with cognitive deficits which prevents consultations and patients who are included in ongoing research prohibiting additional research participation. A diagram showing the flow of participants through each stage of the randomised trial will be made (figure 1).

\section{Experimental intervention}

The experimental intervention is a comprehensive sexual rehabilitation programme. Sexual rehabilitation in this trial is defined as: a time-bound planned process with clear goals and means. Sexual rehabilitation is a process where several actors, including the patient, are working towards regaining improved sexual functioning and coping ability according to their sexual function. The comprehensive sexual rehabilitation programme contains a physical exercise component, including training of the pelvic floor and a psychoeducational component.

\section{The physical components}

Physical exercise

The goal of physical exercise is to achieve an improvement in the patient's physical work capacity, and to eliminate the fear and uncertainty the patient may feel in relation to sexual activity as a form of physical activity. The physical exercise intervention is based on The European Society of Cardiology recommendations for physical activity for cardiovascular patients. ${ }^{54}$ The European Society of Cardiology recommends that all adults promote and maintain their fitness, muscle strength, flexibility and bone health several hours a week. Training must be of high intensity and of 30 min duration. ${ }^{54}$ Furthermore, the intervention is supported by European recommendations for physical training in cardiac patients ${ }^{55}$ and has been tested in two large rehabilitation trials, COPE-ICD and DANREHAB ${ }^{56}{ }^{57}$ A professional physiotherapist with specific knowledge of cardiac rehabilitation initiates the physical exercise programme. Together with the patient, the physiotherapist plans and prepares a physical exercise protocol, taking into account the patient's clinical condition and physical abilities. Sixty minutes is allocated for the initial consultation and preparation of individual training protocol, including pelvic floor exercise instructions.

Physical exercise is initiated at a physiotherapistsupervised setting at the Heart Centre, Rigshospitalet. Using wireless electrodes integrated into t-shirts (Corus-Fit, CardioCardio and Corus Exercise Assistant, V.2.0.16, Finland) potential cardiac arrhythmias, ECG abnormalities such as ST segment changes, T wave alterations, atrial or ventricular arrhythmias and training intensity levels are monitored. The training is initiated with two to three mandatory exercise sessions at Rigshospitalet. Subsequently, the patients can choose to continue the intensive physical exercise regimen either at Rigshospitalet, or at a local CopenHeart-certified facility, supervised by physiotherapists, or as supervised home-based training. Supervised home-based physical training has previously shown similar results to hospital-based training. ${ }^{58}$ This finding has been confirmed in a Danish setting. ${ }^{59}$

One session is structured with 10 min warm-up bicycling, 20 min bicycling with increased intensity (cardiovascular training), 20 min strength exercises and 10 min stretching and cool-down period. The warm-up session is performed at the intensity of $11-12$ on the Borg scale. ${ }^{60}$ The $20 \mathrm{~min}$ cardiovascular training is performed as interval training. Each session is divided into three sections. Each section contains an intensity of 13-17 on the Borg scale and time limit (2-15 min) varying between each section; the second section with longest and highest intensity. A cool-down period of $5 \mathrm{~min}$ is included after $20 \mathrm{~min}$ of cardiovascular training. The strength and strength-related exercises primarily target lower body muscles, and comprise the following four exercises: (1) heel rise performed by repetitions of maximal flexion from standing position; (2) step-up by using a step bench of $27 \mathrm{~cm}$; (3) leg press standardised, starting with $90^{\circ}$ flexion, hyperextension not accepted; (4) $90^{\circ}$ pull-down performed in a cable machine to target abdominal muscles. For step-ups and heel-rises, weight load is calculated as a percentage of body weight (5-20\%) and increased throughout the 12 weeks. Load for leg press is estimated from repetition maximum (RM) testing and increases from $60 \%$ to $70 \%$ of $1 \mathrm{RM}$ during 12 weeks of 
Figure 1 Flow chart.

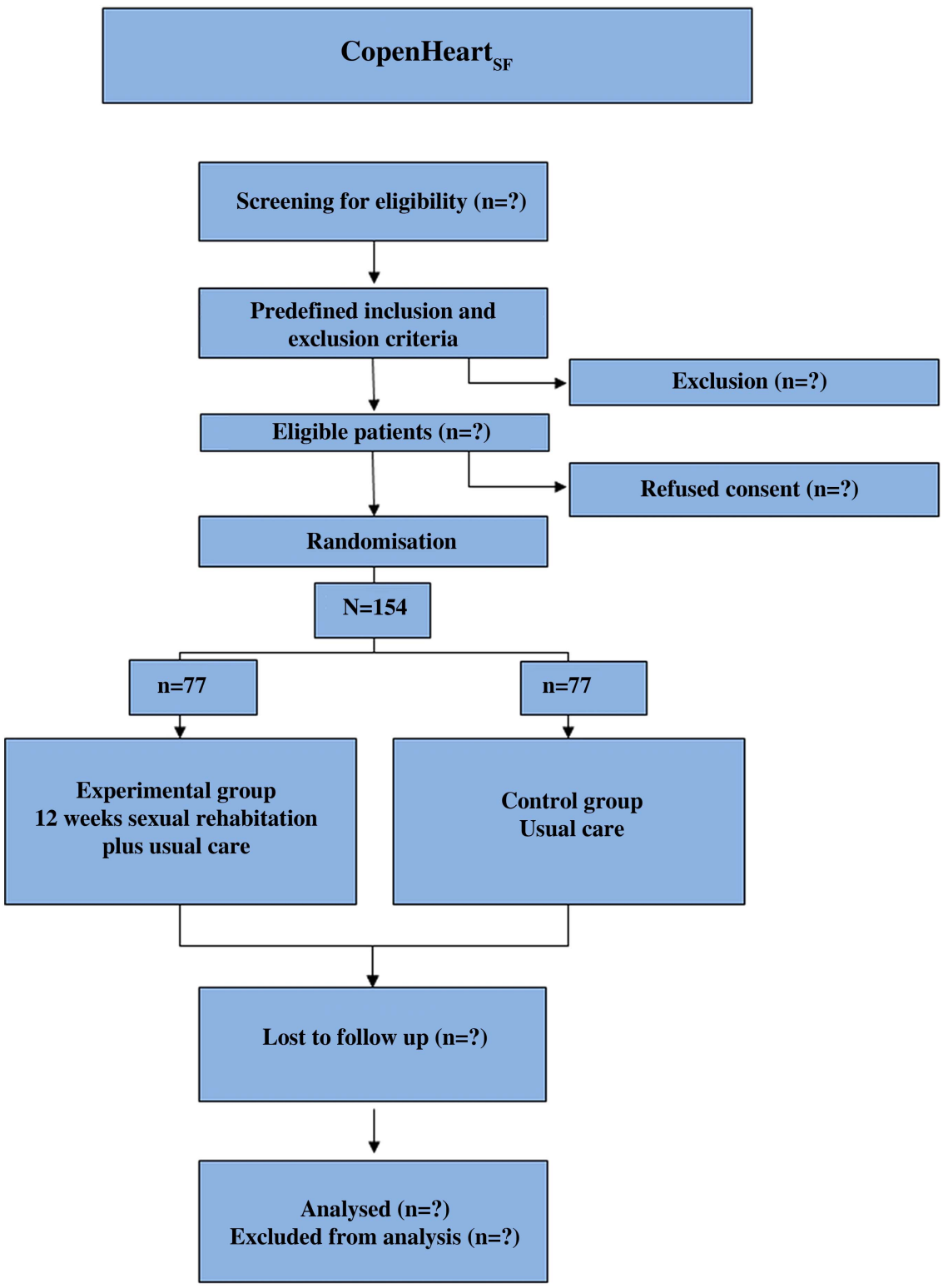

training. All exercises are initiated by $2 \times 12$ repetitions and increased through the programme according to standard guidelines for strength training. ${ }^{61}$

To achieve cardiovascular adjustment the training begins with a warming-up period and ends with a cooldown period. This cardiovascular adjustment has been shown to reduce the risk of ischaemia and arrhythmia in connection to physical exercise. ${ }^{44}$ Participants must mainly exercise in an upright position to decrease left ventricular filling pressure and risk of ischaemia or heart-failure-triggered ventricular arrhythmias. ${ }^{62}$

\section{Pelvic floor exercise}

The bulbocavernosus muscle and the ischiocavernosus muscle, two superficial pelvic floor muscles, are active during erection and enhance rigidity. The bulbocavernosus muscle encircles $33-50 \%$ of the base of the penis. ${ }^{41}$ The pelvic floor training regimen is inspired by Dorey et $a l,{ }^{63}$ who have developed a training regimen for male patients for use in randomised clinical trials. The regiment is developed and tested in a different patient population, and we have therefore modified it to fit cardiovascular patients. Patients are instructed in pelvic floor exercises by a skilled physiotherapist. Patients are instructed to perform their pelvic floor exercises twice daily. Studies showed that a few strong or maximum contractions are more effective when it comes to gaining muscle hypertrophy than several less strong contractions. ${ }^{63}$ Patients are instructed to tighten their pelvic floor muscles as strongly as possible (as if to prevent flatus from escaping) three times when lying, three times when sitting and three times when standing. The duration of the contraction is up to $10 \mathrm{~s}$ each, and patients are informed to have a $10 \mathrm{~s}$ break between each contraction. The physiotherapist instructs the patients on how to contract the bulbocavernosus and ischiocavernosus muscles. In order to ensure that the right muscles are involved, attention is placed on the 
ability to lift the scrotum and retract the penis. To obtain some degree of pelvic floor muscle endurance, the patients are encouraged to tighten the pelvic floor muscles when walking.

To encourage adherence and monitor compliance pulse watches (Polar watch) with extended memory and exercise training logs are handed out. A training log contains information about physical exercise as well as pelvic floor exercise. At the end of the intervention the training $\log$ and the pulse watch are returned and compliance and intensity level are coded independently.

\section{The psychoeducational components}

The goal of the psychoeducative intervention is that the patient learns to interpret and react to relevant physical and psychological symptoms, learns to cope with anxiety and fear, including strategies to manage depressive symptoms and the ability to be sexually active without fear.

A specially trained nurse is responsible for the psychoeducative intervention. The intervention takes a theoretical basis of the patient-centred approach where the emphasis is on support and education. The conversations are based on a holistic view of the patient and focus on the handling of life and managing sexual dysfunction. The intervention is targeted at the modifiable parameters that are reported to affect sexual dysfunction. The psychoeducative intervention is inspired by RR Parse's 64 'Human Becoming Practice Methodologies' three dimensions which can be described as (1) discuss and give meaning to the past, present and future; (2) explore and discuss events and opportunities and (3) pursue imagined possibilities. According to this theory, there are three ways to alter its perceived health: creative ideas, see, hear and feel how a situation could be if it was lived in a different way; recognising personal patterns and value priorities and shed light on the paradoxes by looking at incongruence in a situation and change the view of reality. The nurse is 'truly present' in the process through discussion, quiet contemplation and reflection. The psychoeducative intervention plus physical exercise was tested in the COPE-ICD trial, with positive effects on psychological well-being (mental health) and the general health subscale of the Short Form-36. ${ }^{56}$ The nurse is trained in the psychoeducative conversation through teaching and supervision of nurses who have experience with the 'Human Becoming Practice Methodology' from the COPE-ICD trial. It is based on the theoretical literature that forms the basis for understanding the processes of practice methodology and existing specialty specific knowledge about heart disease, related symptoms and sexology. The supervisor observes and provides feedback in relation to the methods and goals of the conversation. The emphasis is on openness in the interviews and on the nurse's ability to: be silently present while the patient talks, ask questions that encourage reflection, let the patient find answers and solutions and contribute with knowledge, provide advice and guidance when requested and relevant. The training of the nurse takes place prior to the intervention. In practice the intervention will be handled by one nurse with several years of experience working with cardiac patients and trained in sexology. The sexology experience is gained in a 2-week intensive course on basic and clinical sexology including training in sexual therapy. Supervision from a sexologist is available during the intervention. The nurse will conduct consultations with patients individually, and patients are informed that they are welcome to bring spouses/relatives. The consultation will take place in a quiet room in an outpatient setting and last for $45 \mathrm{~min}$. An inspirational guide will form the basis for the consultations. The guide consists of several elements and issues (medical, psychosocial, educational and sexual) that work as inspiration (see box 1).

\section{Usual care}

Participants in the experimental group and in the control group will receive the usual care according to current guidelines. Usual care is, for patients for whom it is not contraindicated, treatment with PDE-5 inhibitors. Patients who are candidates for PDE- 5 inhibitors are encouraged to contact their general practitioner in order to establish the treatment. Use of PDE-5 inhibitors will be monitored in both intervention groups. To assess outcome measures, patients in the control group will be asked to complete questionnaires on equal terms with participants in the experimental group. In addition, they will be tested in the form of cardiopulmonary testing (peak $\mathrm{VO}_{2}$ ) and pelvic floor muscle strength and endurance at baseline and at the end of the trial.

\section{Outcomes and data collection}

In order to evaluate the effect of the comprehensive sexual rehabilitation programme numerous data will be collected.

\section{Primary outcome}

Sexual function will be measured by the IIEF questionnaire after 16 weeks and 6 months. IIEF was developed in conjunction with the clinical trial programme for sildenafil, and has since been adopted as the 'gold standard' measure for efficacy assessment in clinical trials of

Box 1 Inspiration guide for psychoeducational consultations

A brief medical history

Actual thoughts and questions regarding their heart disease and sexual function

Sexual dysfunction

Safety issues

Angina or ICD shock

How the sexual problems affect daily live

Provide the patient with recommendations

Relationship 
erectile dysfunction. It has been linguistically validated in 32 languages including Danish and used as a primary outcome in more than 50 clinical trials. ${ }^{34} 4546$ It consists of 15 items including five domains of sexual function: erectile function, orgasmic function, sexual desire, intercourse satisfaction and overall satisfaction. The IIEF meets psychometric criteria for test reliability and validity, and has a high degree of sensitivity and specificity. ${ }^{46}$ The IIEF is self-assessed, which in sexological research is widely used and well acclaimed.

\section{Secondary outcome}

Sexual function is measured by the PAIS-SR sexual relationship domain. ${ }^{47}$ The overall PAIS-SR measure psychosocial adjustment to illness in terms of seven primary domains of adjustment: Health Care Orientation, Vocational Environment, Domestic Environment, Sexual Relationships, Extended Family Relationships, Social Environment and Psychological Distress. Each PAIS/ PAIS-SR item is rated on a 4-point (0 through 3) scale of adjustment, with higher ratings indicating poorer adjustment status. The sexual relationship domain evaluates shifts in the quality of sexual relations due to the current illness or treatment. It consists of six items and the total score ranges from 0 to 18 . Low scores indicate good adjustment, and high scores indicate poor adjustment.

\section{Exploratory outcomes}

A more extensive evaluation of physical, psychological and demographic status over time will be performed. Physical examination will include pelvic floor strength and endurance assessed according to the Modified Oxford grading scheme which is a manual digital examination of the pelvic floor. It is tested and validated and used in several trials. ${ }^{65}{ }^{66}$ Furthermore, physical capacity will be measured by peak $\mathrm{VO}_{2}$ using cardiopulmonary exercise testing (Ergo-Spiro CS-200, Schiller, Switzerland) with measurement of oxygen uptake $\left(\mathrm{VO}_{2}\right)$, heart rate (HR, bpm), ventilation rate $(1 / \mathrm{min})$, ventilation frequency (number/min), respiratory expiration ratio $\left(\mathrm{CO}_{2} / \mathrm{O}_{2}\right.$ in $\left.\%\right)$, blood pressure, physical activity level (METS) and gas exchange $\left(\mathrm{VO}_{2}\right.$ and $\left.\mathrm{VCO}_{2}\right)$ during progressive loading and in the following recovery period. The test is conducted before the training programme initiates. Intensity performed as a ramp protocol (load gradually increases) with the initial work load of $25 \mathrm{~W}$ and increased by $12.5 \mathrm{~W}$ every minute until exhaustion, usually but not always, is where the patient's oxygen uptake reaches a steady state despite additional load. The test follows current standards for cardiopulmonary exercise testing. ${ }^{67}$ The full test procedure is described by Rasmussen $\mathrm{et} a \mathrm{l}^{50}$ Additionally a series of questionnaires, regarding health-related quality of life, anxiety and depression and sexual dysfunction are administered (see table 1).
Table 1 CopenHeartSF-exploratory quantities subjected to post-hoc analysis

\begin{tabular}{|c|c|c|}
\hline Quantity & $\begin{array}{l}\text { Time of } \\
\text { measure }\end{array}$ & $\begin{array}{l}\text { Type of } \\
\text { quantity }\end{array}$ \\
\hline \multicolumn{3}{|l|}{ Demographic } \\
\hline Age, height, weight & Baseline & Continuous \\
\hline $\begin{array}{l}\text { Marital, educational, } \\
\text { occupational status }\end{array}$ & Baseline & Categorical \\
\hline Smoking & Baseline & Binary $(\mathrm{Y} / \mathrm{N})$ \\
\hline \multicolumn{3}{|l|}{ Clinical } \\
\hline Nutritional status (BMI) & Baseline & Continuous \\
\hline NYHA classification & Baseline & Continuous \\
\hline Type of heart disease & Baseline & Categorical \\
\hline $\begin{array}{l}\text { Type of sexual } \\
\text { dysfunction }\end{array}$ & Baseline & Categorical \\
\hline Diabetes mellitus & Baseline & Binary $(\mathrm{Y} / \mathrm{N})$ \\
\hline Level of physical activity & Baseline & Categorical \\
\hline $\begin{array}{l}\text { Level of rehabilitation } \\
\text { offered }\end{array}$ & Baseline & Categorical \\
\hline $\begin{array}{l}\text { PDE-5 inhibitor intake, } \\
\text { Level of activity within }\end{array}$ & $\begin{array}{l}\text { Baseline, } \\
\text { W12, W16, }\end{array}$ & Categorical \\
\hline $\begin{array}{l}\text { the last } 4 \text { weeks, level of } \\
\text { sexual activity }\end{array}$ & M6 & \\
\hline \multicolumn{3}{|l|}{ Para clinical } \\
\hline Cholesterol level & Baseline & Continuous \\
\hline \multicolumn{3}{|l|}{ Functional capacity } \\
\hline Peak $\mathrm{VO}_{2}$ & $\begin{array}{l}\text { Baseline, } \\
\text { W12 }\end{array}$ & Continuous \\
\hline $\begin{array}{l}\text { Pelvic floor strength and } \\
\text { endurance }\end{array}$ & $\begin{array}{l}\text { Baseline, } \\
\text { W12 }\end{array}$ & Continuous \\
\hline Serious adverse events & $\begin{array}{l}\text { W12, W16, } \\
\text { M6 }\end{array}$ & Continuous \\
\hline \multicolumn{3}{|l|}{ Questionnaires } \\
\hline $\begin{array}{l}\text { SF-36 }{ }^{68} \text { HADS }^{69} \\
\text { EQ-5D-5L } \text { FAME }^{71} \text { Sex } \\
\text { after ICD questionnaire }\end{array}$ & $\begin{array}{l}\text { Baseline, } \\
\text { W16, M6 }\end{array}$ & Continuous \\
\hline
\end{tabular}

BMI, body mass index; Eq-5D-5L, EuroQol; FAME, Female Assessment of Male Erectile Function; HADS, Hospital Anxiety and Depression Scale; NYHA, New York Heart Association; PDE-5, phosphodiesterase-5; SF-36, Short Form-36; Y/N, yes/no.

\section{Blinding}

It is not possible to blind the allocated intervention group for the staff and the participants. ${ }^{72}$ All physical testing, data collection and administration will be conducted by blinded staff, however. Statistical analyses and drawing of conclusions from these will also be conducted blinded to the intervention group.

\section{Sample size and power calculations}

We are planning a trial of the continuous response variable IIEF ${ }^{45} 46$ from independent control and experimental participants with one control per experimental participant. In a previous trial the IIEF within each participant group was normally distributed with a SD of 6 points. ${ }^{34}$ If the true difference in the experimental and control means is 3.5 points, we will need to include 77 experimental participants and 77 control participants (total 154 participants) to obtain a power of $95 \%$ 
$(\beta=5 \%)$ and a type 1 error probability of $5 \%$. Using this sample size, an SD of 4 points and an alternative hypothesis of a mean difference of 2 points for the secondary outcome and a type 1 error probability of $5 \%$ the corresponding power for the secondary outcome is found to be $87 \%$.

\section{Study procedure and randomisation}

To achieve our estimated sample size of 154 participants, patients will be identified from the hospital databases. Patients will be selected consecutively. Patients with an implantable cardioverter defibrillator are required to have the device implanted more than 1 year prior to inclusion and patients with ischaemic heart disease 1 year from event and backward. The 1 year limit has been set so that patients are past their rehabilitation if any is provided. Patients will receive the IIEF questionnaire ${ }^{45}$ by mail including a stamped return envelope. Patients with a score $\leq 25$, the accepted cut-off score ${ }^{46}$ on the initial screening, are invited to attend a preliminary interview with the offer to participate in a randomised clinical trial targeting sexual problems. The participant information is sent to the patient along with the invitation. This gives the patient an opportunity to read the material in advance and to prepare possible questions. At the initial interview/ meeting it is determined whether the patient meets the criteria for participating in the trial. If patients are suited and want to participate they will be randomised to a comprehensive sexual rehabilitation programme plus usual care versus usual care alone. Stratification will be according to patient group (patients with ischaemic heart disease or implantable cardioverter defibrillator) and age ( $\leq 59$ or $\geq 60$ years) and randomised 1:1 to the experimental group or the control group. Randomisation will be performed centrally by the trial coordination centre, Copenhagen Trial Unit, according to a computer-generated allocation sequence with a variable block size concealed from the investigators. Allocation to the intervention groups is carried out when the investigator calls the Copenhagen Trial Unit. Relevant information (personal identification number, strata, etc) is typed into a computer system, and then the participant will be allocated to an intervention group and awarded a four-digit randomisation number. The investigator then informs the patient of the result and on how to proceed by letter. Thus, neither investigators nor patients or relatives can influence to which group the patients are allocated. For both groups, follow-up assessment will take place after 12 weeks (only physical evaluation), 16 weeks and 6 months. Questionnaires will be completed electronically in the questionnaire system Enalyzer with 'single user', which meets the data legislation for logging. At inclusion, the trial participant will receive an email with a link to a website through which questionnaires can be completed. The email contains a login and password for the trial participant's personal access. The participant has the opportunity to go through the website http://www.copenheart.org and enter with the login and password. If patients do not complete the questionnaire electronically, the material can be sent in paper form and an independent trial personnel then enters the responses into the database. Thus data management is handled independently from the researchers who interpret the data. All data are stored electronically in a coded database, and in an independent spread sheet, only accessible for the CopenHeart group. The recruitment process will continue until the number of 154 has been reached.

\section{Statistical analysis}

\section{Analysis of primary and secondary outcomes}

The analysis will be performed according to the intention-to-treat analysis with two-sided significance tests at the 0.05 level. Both outcomes (and outcomes subjected to exploratory analyses) will be analysed using the same procedure. First, we will test if there is an immediate effect of the intervention on the outcome and/or a difference in the response to the intervention between the two patient groups (patients with ischaemic heart disease and patients with implantable cardioverter defibrillator) using model 1 below. Then the follow-up data will be included in the analysis and the long-term effect will be studied using model 2 .

\section{Models and analytical techniques}

Model 1 The equation (equation 1) showing the dependent variable $\mathrm{Y}$ (the outcome) as a function of covariates used in the analysis of the immediate effect of the intervention on the primary outcome is

$$
\mathrm{Y}=\text { intercept }+\mathrm{a} \mathrm{Y}_{\text {baseline }}+\mathrm{bI}+\mathrm{cG}+\mathrm{dI}: \mathrm{G}
$$

$\mathrm{Y}_{\text {baseline }}$ is the baseline value of the outcome, I the indicator of intervention, $G$ the indicator of patient group, and a through $d$ are coefficients to be estimated. The term dI:G stands for interaction between the two covariates $I$ and $G$. If the term bI is significant (the coefficient b differs significantly from 0 ) there is an effect of the intervention common for the two patient groups (ischaemic patients and patients with implantable cardioverter defibrillator). If the term dI:G is significant there is an additional effect of the intervention in one of the two patient groups; thus a subgroup analysis is warranted. In the analysis of the data the univariate general linear model is used. The analysis of the primary outcome is the primary analysis. The subgroup analysis and the analysis of the secondary and of other outcomes should be considered exploratory.

Model 2 In the analysis of follow-up data the time $\mathrm{T}$ (Y is measured 16 weeks and 6 months following randomisation) is included and the mixed model for repeated measures is used. The equation (equation 2) for the fixed effect in this model showing $\mathrm{Y}$ as a function of the covariates is

$$
\begin{aligned}
\mathrm{Y}= & \text { intercept }+\mathrm{a} \mathrm{Y}_{\text {baseline }}+\mathrm{bG}+\mathrm{cI} \\
& +\mathrm{dI}: \mathrm{G}+\mathrm{eT}+\mathrm{fI}: \mathrm{T}+\mathrm{gI}: \mathrm{T}: \mathrm{G}
\end{aligned}
$$


where a through $\mathrm{g}$ are coefficients to be estimated. If the term eT is significant there is a linear trend over time common for both patient groups. If fI:T is significant, this trend is supplemented by an additional trend caused by the intervention and therefore specific for the intervention group. If in addition gI:T:G is significant this added trend differs between the two patient groups (patients with ischaemic heart disease and patients with implantable cardioverter defibrillator). In the mixed model analysis an unstructured covariance matrix will be assumed. If convergence is not attained simpler covariance structure models will be assessed guided by Akaike's criterion or the maximum likelihood test as appropriate.

\section{Missing values}

If the number of missing cases for a given outcome (number of patients with one or more model variables missing) is larger than $5 \%$ or $\mathrm{p}$ of Little's test is $<5 \%$ multiple imputations of the model variables (outcome plus covariates) is performed using SPSS V.17. The range of potential bias in case the missing values should not be random is assessed by doing two imputations (1) imputing missing outcome value in one group by minimum value found in the material and missing outcome value in the other group by maximum value found in material and (2) vice versa. Then in each case an unadjusted analysis is performed to estimate the parameter of interest.

\section{ETHICS AND DISSEMINATION}

The trial complies with the latest Declaration of Helsinki and is registered at ClinicalTrials.gov (NCT01796353). Patients are informed about the trial in writing as well as verbally and only included if a written informed consent is obtained. Patients are assessed in accordance to whether it is safe for them to perform sexual activity. This is carried out according to recommendations from the Princeton consensus group. ${ }^{32} 53$ If patients are suited and want to participate they will be enrolled in the trial. Trial participants are free to withdraw their informed consent at any time and be treated according to the departments' standard treatment procedures. A patient will be withdrawn from the trial if the trial participant withdraws his consent and will, in connection therewith, be informed that termination of the trial will have no implications for his future treatment. Patients who leave the trial will be politely asked for permission to continue to collect data and to use already collected data. If the patient gives permission, he will be included in the final analysis. Only if the patient refuses use of already collected data, will all data relating to him be destroyed. All patient data will be handled and stored in accordance with Danish Data Protection Agency rules and patients are ensured anonymity. The trial will be conducted according to the Act No. 593 of 14 June 2011 on Act on Research Ethics Review of Health Research Projects. The investigator will immediately notify the regional ethics committee if, within the interventions period, there occur serious adverse events, serious adverse reactions or suspected unexpected serious adverse reactions. The report will be accompanied by comments on possible implications for the trial, and notification will be made within 7 days after the investigator has knowledge of the event. The trial has no data monitoring committee however an internal monitor will perform random checks to see if the trial staff work according to the protocol. No risks are anticipated to occur during the sexual rehabilitation programme. As far as we know, there is no previous risk associated with nursing consultations. If the nurse, during the consultation, identifies a need for further consultations with professionals, she will encourage the participant to seek help from the general practitioner, psychologist or in their usual outpatient setting. Risks associated with exercise training and testing are sudden cardiac death associated with ventricular arrhythmias, acute myocardial infarction, and in patients with chronic heart failure, pulmonary oedema and deterioration in left ventricular function. ${ }^{73}$ The last is only found in one study from $1988^{74}$ and has not subsequently been demonstrated in larger studies. ${ }^{75} 76$ In a recent French study of more than 25000 patients with ischaemic heart disease, one-third with chronic heart failure found the risk of cardiac complications at 1:8500 exercise testing and 1:50000 patient exercise hours. ${ }^{77}$ Increasing exercise intensity and age are risk indicators. Therefore, the training intensity will be conducted as moderate high intensity (less than $80 \%$ of $\mathrm{VO}_{2}$ max). To achieve cardiovascular adjustment exercise training as well as testing begins with a warming-up period and ends with a cool-down period, with a gradual downward adjustment of exercise intensity and HR, rather than an abrupt end. This cardiovascular adjustment has been shown to reduce the risk of ischaemia and arrhythmia in connection with physical exercise. ${ }^{42}$ Participants must mainly exercise in an upright position to decrease left ventricular filling pressure and risk of ischaemia or heart failure triggered ventricular arrhythmias. When these precautions are respected, exercise training as well as exercise testing are considered to possess a low risk for the participants. There is, as far as we know, no previously known risk associated with pelvic floor exercise. Testing or examination of the pelvic floor may be associated with discomfort for the participants but is not considered to be associated with any risk. Staff members will be trained according to guidelines to handle any emergencies.

\section{Dissemination plan}

Positive, neutral and negative results of the trial will be submitted to international peer reviewed journals of nursing, cardiology or sexology. Furthermore, results will be presented at national and international conferences relevant to subject fields. Authorship will be allocated using the guidelines for authorship defined by the International Committee of Medical Journal Editors and depends on the personal involvement. All the articles, 
abstracts as well as the results will be posted on the website http://www.copenheart.org. The website will be continuously updated and will be highlighted through the scientific articles. CopenHeart staff will have access to data. Ethic committees and competent authorities will be able to obtain direct access to data and documentation.

\section{DISCUSSION}

This randomised clinical trial testing the effect of a comprehensive sexual rehabilitation intervention on a population of patients with implantable cardioverter defibrillator or patients with ischaemic heart disease seems to be the first one in its field. The trial is expected to contribute with results that can improve patients' problems related to heart disease and sexual function. Additionally, it is believed that the trial can provide a systematic approach that may 1 day inform national consensus on how to treat sexual dysfunction in heart patients. Furthermore, the results of the trial are expected to contribute to the international debate on sexual rehabilitation of patients with heart disease.

The trial is designed with central stratified randomisation which secures against selection bias. ${ }^{78}$ 79 The primary outcome is assessed blinded to intervention and so are all statistical analysis, which should reduce detection and interpretation bias. ${ }^{78} 79$

\section{Trajectory}

Inclusion was initiated on February 2013 and is expected to continue until June 2014.

\section{Author affiliations \\ ${ }^{1}$ Department of Cardiology, Copenhagen University Hospital, Bispebjerg Hospital, Copenhagen, Denmark \\ ${ }^{2}$ The Heart Centre, Copenhagen University Hospital, Rigshospitalet, Copenhagen, Denmark \\ ${ }^{3}$ National Institute of Public Health, University of Southern Denmark, Denmark ${ }^{4}$ Faculty of Health Science, Institute of Clinical Medicine, University of Copenhagen, Copenhagen, Denmark \\ ${ }^{5}$ The Copenhagen Trial Unit, Centre for Clinical Intervention Research, Rigshospitalet, Copenhagen University Hospital, Copenhagen, Denmark ${ }^{6}$ Sexological Clinic, Psychiatric Center Copenhagen, Copenhagen University Hospital, Rigshospitalet, Copenhagen, Denmark \\ ${ }^{7}$ Wichita State University, Wichita, Kansas, USA \\ ${ }^{8}$ Department of Social and Welfare Studies, Linköping University, Linköping, Sweden}

Acknowledgements The test and rehabilitation team responsible for the trial is Karina Jensen, Lars Tang, Helena Tjalk Sørensen, Signe Gils and Katrine Tingholm Erhardsen.

Contributors PW specifically designed the statistical analysis plan. PPJ, SKB and A-DZ drafted the manuscript. All authors designed the study and developed the protocol, revised the manuscript critically and have given their final approval of the version to be published.

Funding The CopenHeartSF trial has received funding from: The Danish Heart Foundation (no. 13-04-R95-A4669-22744); The Health Foundation (no. 2013B208); Danish Council for Strategic Research (no. 10-092790) and The Danish Nursing Council.

Competing interests None.
Ethics approval Trial protocol has been approved by the Regional Ethics Committee (no H-4-2012-168) and the Danish Data Protection Agency (no 2007-58-0015).

Provenance and peer review Not commissioned; peer reviewed for ethical and funding approval prior to submission.

Open Access This is an Open Access article distributed in accordance with the Creative Commons Attribution Non Commercial (CC BY-NC 3.0) license, which permits others to distribute, remix, adapt, build upon this work noncommercially, and license their derivative works on different terms, provided the original work is properly cited and the use is non-commercial. See: http:// creativecommons.org/licenses/by-nc/3.0/

\section{REFERENCES}

1. Hoekstra T, Jaarsma T, Sanderman R, et al. Perceived sexual difficulties and associated factors in patients with heart failure. Am Heart J 2012;163:246-51.

2. Laumann EO, Paik A, Rosen RC. Sexual dysfunction in the United States: prevalence and predictors. JAMA 1999;281:537-44.

3. Feldman HA, Goldstein I, Hatzichristou DG, et al. Impotence and its medical and psychosocial correlates: results of the Massachusetts Male Aging Study. J Urol 1994;151:54-61.

4. Feldman HA, Johannes CB, Derby CA, et al. Erectile dysfunction and coronary risk factors: prospective results from the Massachusetts male aging study. Prev Med 2000;30:328-38.

5. Bacon CG, Mittleman MA, Kawachi I, et al. Sexual function in men older than 50 years of age: results from the health professionals follow-up study. Ann Intern Med 2003;139:161-8.

6. Levine GN, Steinke EE, Bakaeen FG, et al. Sexual activity and cardiovascular disease: a scientific statement from the American Heart Association. Circulation 2012;125:1058-72.

7. Steinke EE. Sexual dysfunction in women with cardiovascular disease: what do we know? J Cardiovasc Nurs 2010;25:151-8.

8. Lewis RW, Fugl-Meyer KS, Corona G, et al. Definitions/ epidemiology/risk factors for sexual dysfunction. J Sex Med 2010;7(4 Pt 2):1598-607.

9. [No authors listed]. NIH Consensus Conference. Impotence. NIH Consensus Development Panel on Impotence. JAMA 1993;270:83-90.

10. Rastogi S, Rodriguez JJ, Kapur V, et al. Why do patients with heart failure suffer from erectile dysfunction? A critical review and suggestions on how to approach this problem. Int $J$ Impot Res 2005;17(Suppl 1):S25-36.

11. Berg SK, Elleman-Jensen L, Zwisler AD, et al. Sexual concerns and practices after ICD implantation: findings of the COPE-ICD rehabilitation trial. Eur J Cardiovasc Nurs 2013;12:468-74.

12. Bortolotti A, Parazzini F, Colli E, et al. The epidemiology of erectile dysfunction and its risk factors. Int $J$ Androl 1997;20:323-34.

13. Herbert K, Lopez B, Castellano J, et al. The prevalence of erectile dysfunction in heart failure patients by race and ethnicity. Int $\mathrm{J}$ Impot Res 2008;20:507-11.

14. Kloner RA, Mullin SH, Shook T, et al. Erectile dysfunction in the cardiac patient: how common and should we treat? J Urol 2003;170 (2 Pt 2):S46-50; discussion S50.

15. Montorsi F, Briganti A, Salonia A, et al. Erectile dysfunction prevalence, time of onset and association with risk factors in 300 consecutive patients with acute chest pain and angiographically documented coronary artery disease. Eur Urol 2003;44:360-4; discussion 364-5.

16. Steinke EE. Sexual concerns of patients and partners after an implantable cardioverter defibrillator. Dimens Crit Care Nurs 2003;22:89-96.

17. Dabrowski R, Smolis-Bak E, Kowalik I, et al. Quality of life and depression in patients with different patterns of atrial fibrillation. Kardiol Pol 2010;68:1133-9.

18. Drory $\mathrm{Y}, \mathrm{Kravetz} \mathrm{S}$, Weingarten M. Comparison of sexual activity of women and men after a first acute myocardial infarction. $A m \mathrm{~J}$ Cardiol 2000;85:1283-7.

19. Foroutan SK, Rajabi M. Erectile dysfunction in men with angiographically documented coronary artery disease. Urol $J$ 2007;4:28-32.

20. Justo $\mathrm{D}$, Arbel $\mathrm{Y}$, Mulat $\mathrm{B}$, et al. Sexual activity and erectile dysfunction in elderly men with angiographically documented coronary artery disease. Int J Impot Res 2010;22:40-4.

21. Dunn KM, Croft PR, Hackett Gl. Association of sexual problems with social, psychological, and physical problems in men and women: a cross sectional population survey. J Epidemiol Community Health 1999;53:144-8 
22. Friedman S. Cardiac disease, anxiety, and sexual functioning. $A m \mathrm{~J}$ Cardiol 2000;86:46F-50F.

23. Kriston L, Gunzler C, Agyemang A, et al. Effect of sexual function on health-related quality of life mediated by depressive symptoms in cardiac rehabilitation. findings of the SPARK project in 493 patients. J Sex Med 2010;7:2044-55.

24. Mulat B, Arbel $\mathrm{Y}$, Mashav N, et al. Depressive symptoms and erectile dysfunction in men with coronary artery disease. Urology 2010;75:104-7.

25. Makhlouf A, Kparker A, Niederberger CS. Depression and erectile dysfunction. Urol Clin North Am 2007;34:565-74, vii.

26. Roose SP. Depression: links with ischemic heart disease and erectile dysfunction. $J$ Clin Psychiatry 2003:64(Suppl 10):26-30.

27. Dickstein K, Cohen-Solal A, Filippatos G, et al. ESC Guidelines for the diagnosis and treatment of acute and chronic heart failure 2008: the Task Force for the Diagnosis and Treatment of Acute and Chronic Heart Failure 2008 of the European Society of Cardiology. Developed in collaboration with the Heart Failure Association of the ESC (HFA) and endorsed by the European Society of Intensive Care Medicine (ESICM). Eur Heart J 2008;29:2388-442.

28. Heart Failure Society of America. HFSA 2006 Comprehensive Heart Failure Practice Guideline. J Card Fail 2006;12:e1-2.

29. Bedell SE, Duperval M, Goldberg R. Cardiologists' discussions about sexuality with patients with chronic coronary artery disease. Am Heart J 2002;144:239-42.

30. Jaarsma T, Stromberg A, Fridlund B, et al. Sexual counselling of cardiac patients: nurses' perception of practice, responsibility and confidence. Eur J Cardiovasc Nurs 2010;9:24-9.

31. Jackson G, Boon N, Eardley I, et al. Erectile dysfunction and coronary artery disease prediction: evidence-based guidance and consensus. Int J Clin Pract 2010;64:848-57.

32. Jackson G, Rosen RC, Kloner RA, et al. The second Princeton consensus on sexual dysfunction and cardiac risk: new guidelines for sexual medicine. J Sex Med 2006;3:28-36; discussion 36.

33. Gupta BP, Murad MH, Clifton MM, et al. The effect of lifestyle modification and cardiovascular risk factor reduction on erectile dysfunction: a systematic review and meta-analysis. Arch Intern Med 2011:171:1797-803.

34. Maio G, Saraeb S, Marchiori A. Physical activity and PDE5 inhibitors in the treatment of erectile dysfunction: results of a randomized controlled study. J Sex Med 2010;7:2201-8.

35. Roviaro R, Holmes D, Holmsten R. Influence of a cardiac rehabilitation program on the cardiovascular, psychological, and social functioning of cardiac patients. J Behav Med 1984;7:61.

36. Froelicher ES, Kee LL, Newton KM, et al. Return to work, sexual activity, and other activities after acute myocardial infarction. Heart Lung 1994;23:423-35.

37. Belardinelli $R$, Lacalaprice $F$, Faccenda $E$, et al. Effects of short-term moderate exercise training on sexual function in male patients with chronic stable heart failure. Int J Cardiol 2005;101:83-90.

38. Bertie J, King A, Reed N, et al. Benefits and weaknesses of a cardiac rehabilitation programme. J R Coll Physicians Lond 1992;26:147-51.

39. Lidell E, Fridlund B. Long-term effects of a comprehensive rehabilitation programme after myocardial infarction. Scand $J$ Caring Sci 1996;10:67-74.

40. Klein R, Bar-on E, Klein J, et al. The impact of sexual therapy on patients after cardiac events participating in a cardiac rehabilitation program. Eur J Cardiovasc Prev Rehabil 2007;14:672-8.

41. Dorey G, Speakman MJ, Feneley RC, et al. Pelvic floor exercises for erectile dysfunction. BJU Int 2005;96:595-7.

42. Van Kampen M, De Weerdt W, Claes H, et al. Treatment of erectile dysfunction by perineal exercise, electromyographic biofeedback, and electrical stimulation. Phys Ther 2003;83:536-43.

43. Balady GJ, Williams MA, Ades PA, et al. Core components of cardiac rehabilitation/secondary prevention programs: 2007 update: a scientific statement from the American Heart Association Exercise, Cardiac Rehabilitation, and Prevention Committee, the Council on Clinical Cardiology; the Councils on Cardiovascular Nursing, Epidemiology and Prevention, and Nutrition, Physical Activity, and Metabolism; and the American Association of Cardiovascular and Pulmonary Rehabilitation. J Cardiopulm Rehabil Prev 2007;27:121-9.

44. Fitchet A, Doherty PJ, Bundy C, et al. Comprehensive cardiac rehabilitation programme for implantable cardioverter-defibrillator patients: a randomised controlled trial. Heart 2003;89:155-60.

45. Rosen RC, Riley A, Wagner G, et al. The international index of erectile function (IIEF): a multidimensional scale for assessment of erectile dysfunction. Urology 1997;49:822-30.
46. Rosen RC, Cappelleri JC, Gendrano N III. The International Index of Erectile Function (IIEF): a state-of-the-science review. Int J Impot Res 2002;14:226-44.

47. Derogatis LR. The psychosocial adjustment to illness scale (PAIS). $J$ Psychosom Res 1986;30:77-91.

48. Westlake C, Dracup K, Walden JA, et al. Sexuality of patients with advanced heart failure and their spouses or partners. $J$ Heart Lung Transplant 1999;18:1133-8.

49. Jaarsma T. Sexual problems in heart failure patients. Eur $J$ Cardiovasc Nurs 2002;1:61-7.

50. Rasmussen TB, Zwisler AD, Sibilitz KL, et al. A randomised clinical trial of comprehensive cardiac rehabilitation versus usual care for patients treated for infective endocarditis-the CopenHeartIE trial protocol. BMJ Open 2012:2:10

51. Risom SS, Zwisler AD, Rasmussen TB, et al. The effect of integrated cardiac rehabilitation versus treatment as usual for atrial fibrillation patients treated with ablation: the randomised CopenHeartRFA trial protocol. BMJ Open 2013;3:10.

52. Sibilitz KL, Berg SK, Hansen TB, et al. Effect of comprehensive cardiac rehabilitation after heart valve surgery (CopenHeartVR): study protocol for a randomised clinical trial. Trials 2013;14:104.

53. Nehra A, Jackson G, Miner M, et al. The Princeton III Consensus recommendations for the management of erectile dysfunction and cardiovascular disease. Mayo Clin Proc 2012;87:766-78.

54. Perk J, De Backer $\mathrm{G}$, Gohlke $\mathrm{H}$, et al. European guidelines on cardiovascular disease prevention in clinical practice (version 2012) the fifth joint task force of the European society of cardiology and other societies on cardiovascular disease prevention in clinical practice (constituted by representatives of nine societies and by invited experts). Int J Behav Med 2012;19:403-88.

55. Piepoli MF, Corra U, Benzer W, et al. Secondary prevention through cardiac rehabilitation: from knowledge to implementation. A position paper from the Cardiac Rehabilitation Section of the European Association of Cardiovascular Prevention and Rehabilitation. Eur $J$ Cardiovasc Prev Rehabil 2010;17:1-17.

56. Berg SK. Comprehensive rehabilitation for patients with ICD $[\mathrm{PhD}$ dissertation]. [Aarhus]. Faculty of Health Sciences. Aarhus University, 2011.

57. Zwisler AD, Soja AM, Rasmussen S, et al. Hospital-based comprehensive cardiac rehabilitation versus usual care among patients with congestive heart failure, ischemic heart disease, or high risk of ischemic heart disease: 12-month results of a randomized clinical trial. Am Heart J 2008;155:1106-13.

58. Ashworth NL, Chad KE, Harrison EL, et al. Home versus center based physical activity programs in older adults. Cochrane Database Syst Rev 2005;(1):CD004017.

59. Oerkild B, Frederiksen M, Hansen JF, et al. Home-based cardiac rehabilitation is as effective as centre-based cardiac rehabilitation among elderly with coronary heart disease: results from a randomised clinical trial. Age Ageing 2011;40:78-85.

60. Borg GA. Psychophysical bases of perceived exertion. Med Sci Sports Exerc 1982:14:377-81.

61. Williams MA, Haskell WL, Ades PA, et al. Resistance exercise in individuals with and without cardiovascular disease: 2007 update: a scientific statement from the American Heart Association Council on Clinical Cardiology and Council on Nutrition, Physical Activity, and Metabolism. Circulation 2007:116:572-84.

62. Lampman RM, Knight BP. Prescribing exercise training for patients with defibrillators. Am J Phys Med Rehabil 2000;79:292-7.

63. Dorey G, Glazener C, Buckley B, et al. Developing a pelvic floor muscle training regimen for use in a trial intervention. Physiotherapy 2009;95:199-209.

64. Parse RR. The human becoming school of thought: a perspective for nurses and other health professionals. Thousand Oaks, CA: Sage, 1998.

65. Tibaek S, Klarskov P, Lund Hansen B, et al. Pelvic floor muscle training before transurethral resection of the prostate: a randomized, controlled, blinded study. Scand J Urol Nephrol 2007;41:329-34.

66. Schüssler B. Pelvic floor re-education: principles and practice. London: Springer, 1994.

67. Mezzani A, Agostoni P, Cohen-Solal A, et al. Standards for the use of cardiopulmonary exercise testing for the functional evaluation of cardiac patients: a report from the Exercise Physiology Section of the European Association for Cardiovascular Prevention and Rehabilitation. Eur J Cardiovasc Prev Rehabil 2009;16:249-67.

68. Ware JE, Kosinski M, Gandek B. SF-36 Health Survey. Manual and interpretation guide 2005. Boston, MA: The Health Institute, New England Medical Center.

69. Zigmond AS, Snaith RP. The hospital anxiety and depression scale. Acta Psychiatr Scand 1983;67:361-70. 
70. Drummond MF. Methods for the economic evaluation of health care programmes. 3rd edn. Oxford: Oxford University Press, 2005.

71. Rubio-Aurioles E, Sand M, Terrein-Roccatti N, et al. Female Assessment of Male Erectile dysfunction detection scale (FAME): development and validation. J Sex Med 2009;6:2255-70.

72. Boutron I, Moher D, Altman DG, et al. Extending the CONSORT statement to randomized trials of nonpharmacologic treatment: explanation and elaboration. Ann Intern Med 2008;148:295-309.

73. Fletcher GF, Balady GJ, Amsterdam EA, et al. Exercise standards for testing and training: a statement for healthcare professionals from the American Heart Association. Circulation 2001;104:1694-740.

74. Jugdutt BI, Michorowski BL, Kappagoda CT. Exercise training after anterior $Q$ wave myocardial infarction: importance of regional left ventricular function and topography. J Am Coll Cardiol 1988;12:362-72.

75. Giannuzzi P, Tavazzi L, Temporelli PL, et al. Long-term physical training and left ventricular remodeling after anterior myocardial infarction: results of the Exercise in Anterior Myocardial Infarction (EAMI) trial. EAMI Study Group. J Am Coll Cardiol 1993;22:1821-9.

76. Otsuka $\mathrm{Y}$, Takaki $\mathrm{H}$, Okano $\mathrm{Y}$, et al. Exercise training without ventricular remodeling in patients with moderate to severe left ventricular dysfunction early after acute myocardial infarction. Int $J$ Cardiol 2003;87:237-44.

77. Pavy B, lliou MC, Meurin P, et al. Safety of exercise training for cardiac patients: results of the French registry of complications during cardiac rehabilitation. Arch Intern Med 2006;166:2329-34.

78. Savovic J, Jones HE, Altman DG, et al. Influence of reported study design characteristics on intervention effect estimates from randomized, controlled trials. Ann Intern Med 2012;157:429-38.

79. Wood L, Egger M, Gluud LL, et al. Empirical evidence of bias in treatment effect estimates in controlled trials with different interventions and outcomes: meta-epidemiological study. BMJ 2008;336:601-5. 


\section{Correction}

Johansen PP, Zwisler A-D, HastrupSvendsen J, et al. The CopenHeartSF trialcomprehensive sexual rehabilitation programme for male patients with implantable cardioverter defibrillator or ischaemic heart disease and impaired sexual function: protocol of a randomised clinical trial. BMJ Open 2013;3:e003967.

There has been an update to the Statistical Analyses Plan. The new Statistical Analysis section should read:

\section{Statistical analysis}

The analysis will follow the intention-to-treat principle with two-sided significance test at the 5\% level. Continuous outcomes will follow the same procedure as described in the following for the primary outcome. The primary outcome is the International Index of Erectile Function overall score. The five domains of the questionnaire are all exploratory outcome, but particular attention is given the Erectile Function domain. The secondary outcome is PAIS-SR sexual relationship domain.

The explorative physical outcomes are pelvic floor strength and endurance (one categorical and two continuous variables), peak VO2, heart rate (beats per minute), blood pressure, Watt Max, Anaerobic Threshold, and VE/VCO2 slope. The questionnaire-based exploratory outcomes are SF-36 (the two component scores: physical (SF36-PCS) and mental (SF36-MCS)), Hospital Anxiety and Depression Scale (HADS) anxiety and depression (binary variable: score of 8+) and EQ-5D-5L converted to index score. Sex after ICD-questionnaires (reported as categorical variables) are evaluated for ICD patients.

The primary model for assessing the effect of intervention is the univariate general linear model. This model assesses (1) whether there is an effect of the intervention 16 weeks after randomization, between the intervention group and the control group. If there is a statistically significant effect we will perform subgroup analysis and test (2) whether there is a difference between the two patient groups regarding the size of the effect.

Model 2 includes the follow-up data (month six) using a mixed model because of repeated outcome measures. In this model the baseline value of the outcome, intervention indicator (I), patients indicator $(G)$, the interaction between $I$ and $G$ and stratification variable (aged above and below 60 years) are included.

Subgroup analysis of the primary outcome and all analyses of the secondary and exploratory outcomes are considered hypothesis generating if the effects are statistically significant $(\mathrm{P}<0.05)$.

If missing values of the primary outcome is above $15 \%$ or the P-value of Little's test is below 0.05 multiple imputation techniques will be used. If the intervention effect of the primary analysis in the univariate general linear model is significant, the analysis is supplemented with a worst/best case analysis. The results of the multiple imputed dataset are considered the primary analysis.

Open Access This is an Open Access article distributed in accordance with the Creative Commons Attribution Non Commercial (CC BY-NC 4.0) license, which permits others to distribute, remix, adapt, build upon this work noncommercially, and license their derivative works on different terms, provided the original work is properly cited and the use is non-commercial. See: http://creativecommons.org/licenses/by-nc/4.0/

BMJ Open 2017;7:e003967corr1. doi:10.1136/bmjopen-2013-003967corr1

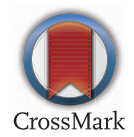

